\title{
Invasive pneumococcal infection in a man with hyposplenism
}

\author{
Satoshi Yoshimura MD, Han-Seung Yoon MD PhD \\ — Cite as: CMAJ 2018 September 10;190:E1084. doi: 10.1503/cmaj.180336
}

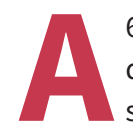

60-year-old man presented to the emergency department with fever, dyspnea and ecchymosis on his trunk and face that had spread rapidly to his entire body within six hours, after one day of malaise (Figure 1A). He had severe acute epiglottitis five years previously, and cellulitis and appendicitis in childhood. He had no history of splenectomy or hyposplenism and had never received a pneumococcal vaccination. There was no evidence of common immunodeficiencies (e.g., hypogammaglobulinemia) or viral infection, but Streptococcus pneumoniae was identified in blood cultures. Urgent computed tomography showed a hypoplastic spleen (Figure 1B, arrow). We diagnosed invasive pneumococcal infection and administered a full dose of linezolid.

Despite treatment, our patient died of multiple organ failure on day 6 of his hospital stay. An autopsy showed marked hypoplasia of the spleen (Figure 1D) and histologic examination showed prominent reduction of white pulp mixed with unremarkable red pulp (Figure 1E). There was also bilateral adrenal hemorrhage (Figure $1 \mathrm{~F}$ ), consistent with Waterhouse-Friderichsen syndrome.

The cause of the hyposplenism in our patient remains unclear, but his case shows that functional hyposplenism, like that occurring after splenectomy, can lead to fatal infection. ${ }^{1,2}$ The risk of invasive pneumococcal infection in patients with splenectomy is more than 50 times higher than in the general population. ${ }^{3}$ The spleen is crucial for production of immunoglobulin M antibodies that opsonize encapsulated pathogens (S. pneumonia, Haemophilus influenzae type b and Neisseria meningitidis). ${ }^{1,2}$

People with hyposplenism require pneumococcal vaccination and prompt treatment with full-dose antibiotics at the onset of infection. ${ }^{1,2}$ Antibiotic prophylaxis should be considered in some cases. ${ }^{1,2} \mathrm{~A}$ history of recurrent or severe infections should raise clinical suspicion for immunodeficiency. ${ }^{2}$ We suggest that
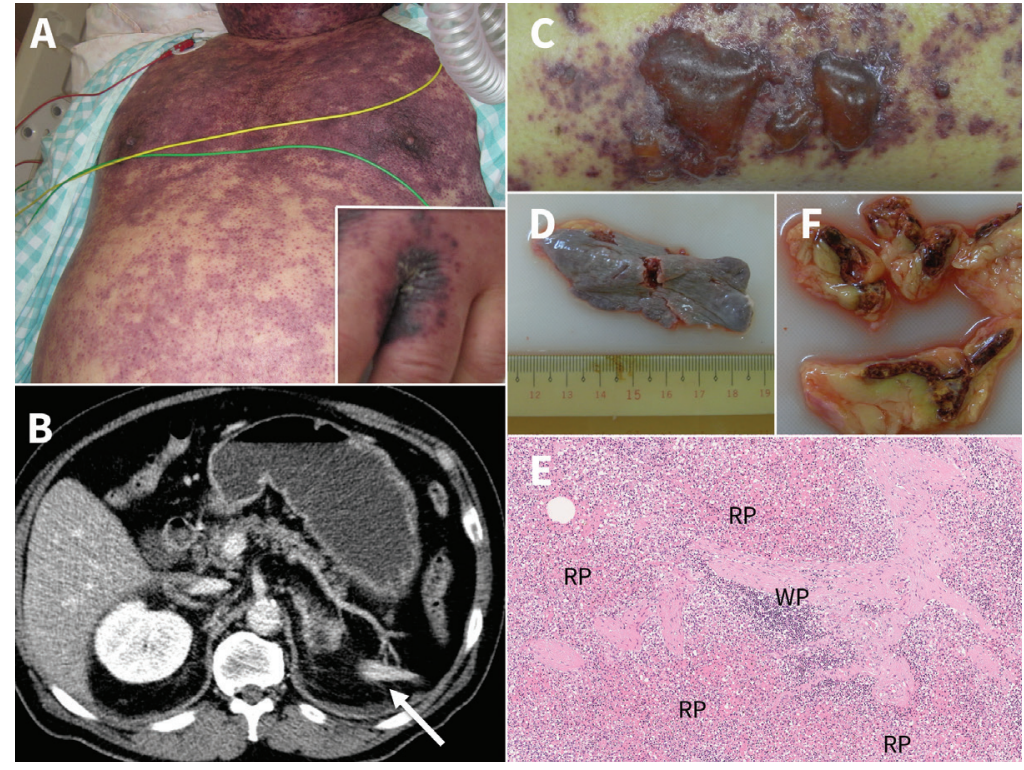

Figure 1: (A) Rapidly spreading systemic gangrene and erythematous rash in a 60-year-old man with hyposplenism (inset: close-up of his left finger). (B) Computed tomographic image showing a small spleen. On autopsy, (C) purpura fulminans with cutaneous vesicles was identified, (D) the spleen was hypoplastic $\left(6 \times 3 \times 1 \mathrm{~cm}^{3}\right.$ in size and weighing $\left.8 \mathrm{~g}\right)$, and (E) the spleen shows numerous severely atrophic areas of white pulp (WP) and unremarkable red pulp (RP). (F) Adrenal hemorrhage was identified on gross examination.

patients with overwhelming infections related to encapsulated organisms should be evaluated for hyposplenism.

\section{References}

1. Bonanni P, Grazzini M, Niccolai G, et al. Recommended vaccinations for asplenic and hyposplenic adult patients. Hum Vaccin Immunother 2017;13: 359-68.

2. Di Sabatino A, Carsetti R, Corazza GR. Post-splenectomy and hyposplenic states. Lancet 2011;378:86-97.

3. Hansen K, Singer DB. Asplenic-hyposplenic overwhelming sepsis: postsplenectomy sepsis revisited. Pediatr Dev Pathol 2001;4:105-21.

\section{Competing interests: None declared.}

This article has been peer reviewed.

The authors have obtained consent from the family.
Affiliations: Department of Cardiology (Yoshimura), Hakujujikai Sasebo Chuo Hospital; Department of Pathology (Yoon), Centre for Digital Pathology Education, Nagasaki University Graduate School of Biomedical Science, Nagasaki, Japan
Acknowledgements: We thank Drs. Yoshihisa Kizaki and Tsutomu Kobayashi for their valuable advice regarding diagnostic strategies, and Dr. Nobuhisa Yonemitsu and Sunao Katafuchi for their advice and support for the autopsy.

Correspondence to: Satoshi Yoshimura, yoshimuras1986@gmail.com 